\title{
Evidence for a New Phase in the Domany-Kinzel Cellular Automaton
}

\author{
M. L. Martins, ${ }^{(a)}$ H. F. Verona de Resende, C. Tsallis, and A. C. N. de Magalhães \\ Centro Brasileiro de Pesquisas Físicas, Conselho Nacional de Desenvolvimento Científico e Tecnológico, \\ Rua Dr. Xavier Sigaud 150, Urca, 22290 Rio de Janeiro, Rio de Janeiro, Brazil
}

(Received 27 November 1990)

\begin{abstract}
We consider a generalized version (including anisotropy) of the stochastic one-dimensional cellular automaton studied by Domany and Kinzel. It recovers Wolfram-like deterministic cellular automata as particular cases. The phase diagram presents three (and not two, as previously suggested) phases which were detected through the numerical study of both the order parameter and the sensitivity to initial conditions. The various universality classes are exhibited as well.

PACS numbers: $87.10 .+\mathrm{e}, 02.50 .+\mathrm{s}, 89.80 .+\mathrm{h}$
\end{abstract}

Cellular automata (CA) are totally discrete dynamical systems (discrete space, discrete time, and discrete number of states) which find general applications in physics, chemistry, biology, computer science, etc. ${ }^{1}$ With each site (noted $i$ ) is associated a variable $\sigma_{i}$ which can be in $k$ different states $\sigma_{i}=0,1, \ldots, k-1$. The dynamics is defined, at each time step, by rules depending on the values at a previous time of $\left\{\sigma_{i}\right\}$ associated with a given number of $r$ arbitrary sites (called inputs). Usually one considers regular lattices and the inputs refer to the sites on the local neighborhood only. The local rules of a CA may be probabilistic or deterministic and the sites are simultaneously updated. Thus we recognize the Glauber dynamics, for example, as a special case of a CA. However, in the more general context of CA, the dynamics is not restricted to the usual Boltzmann weight and detailed balance. Therefore, CA do not necessarily evolve towards equilibrium. Thus they can model chemical reactions, crystal growth models, turbulence, biological problems, or other nonlinear processes far from thermal equilibrium. ${ }^{2}$

Although $d$-dimensional probabilistic CA (PCA) describe processes far from equilibrium, they can be mapped onto $(d+1)$-dimensional statistical-mechanics models. ${ }^{3}$ The corresponding spin model is, in general, anisotropic involving multispin interactions and fields, with coupling constants calculated from the parameters (conditional probabilities) specifying the evolution rule of the PCA. In contrast to equilibrium dynamics even one-dimensional PCA exhibits continuous phase transitions with universal critical exponents and scaling laws.

In this paper we study, by Monte Carlo simulation, the phase diagram and criticality of a generalization of the one-dimensional PCA considered by Domany and Kinzel. ${ }^{4}$ This PCA contains, as a special case, the problem of directed percolation in two dimensions; furthermore, this PCA has been useful for understanding catalysis in chemical reactions. ${ }^{5}$ Specifically our system consists of a one-dimensional chain of $N$ lattice sites $(i=1,2, \ldots, N)$, with periodic boundary conditions. Each site has two possible states $\sigma_{i}=0,1$. The state of the system at time $t$ is specified by the $\left\{\sigma_{i}(t)\right\}$. At the next time step, the state of a given site is $\sigma_{i}(t+1)$ $=0$ or 1 according to the conditional probabilities $\left\{P\left(\sigma_{i-1}(t), \sigma_{i}(t) / \sigma_{i}(t+1)\right)\right\}, \quad$ namely, $\quad P(0,1 / 1) \equiv p_{1}$, $P(1,1 / 1) \equiv p_{2}, P(1,0 / 1) \equiv p_{3}[P(0,0 / 1)=0$ because we consider only legal rules, since this probability acts as an external field conjugated with the order parameter]. Naturally $P\left(\sigma_{i-1}, \sigma_{i} / 0\right)=1-P\left(\sigma_{i-1}, \sigma_{i} / 1\right)$. We extend the PCA considered by $\mathrm{Kinzel}^{4}$ by allowing $p_{1} \neq p_{3}$. In the simulations, we used, for each $\left(p_{1}, p_{2}, p_{3}\right)$ and each $N$, a quite large number (typically up to 50) of random starting configurations where all states were equally probable. Depending on the value of $\left(p_{1}, p_{2}, p_{3}\right)$ the $t \rightarrow \infty$ asymptotic state is homogeneous (with all sites 0 ; frozen phase) or has a finite fraction of interchanging sites with value 1 . This phase transition is continuous and characterized by universal critical exponents.

We define an order parameter $M$ as the $t \rightarrow \infty$ fraction of sites with value 1. In Fig. 1(a) we show the critical surface separating the frozen from the nonfrozen region [the case $p_{1}=p_{3}$ is shown in Fig. 1(b)]. For the $p_{1}=p_{3}$ case our results are consistent with those obtained by $\mathrm{Kinzel}^{4}$ using transfer-matrix and finite-size scaling methods. We can see that the present Monte Carlo simulations indicate that the ordered state is dense enough to support a transition far from the deterministic limit $p_{2}=0, p_{1}=p_{3}=1$. We also calculate the orderparameter critical exponent $\beta\left[M=\left(p_{1}-p_{1 c}\right)^{\beta}\right]$. For $p_{1}=p_{3}$, we find $\beta=0.25 \pm 0.02$, to be compared with Kinzel's value $0.273 \pm 0.002$ (our error bar of 0.02 has been adopted in order to satisfactorily cover all types of fluctuations in all the cases we have run in the computer). Our Monte Carlo simulations support the hypothesis of universality, i.e., the same critical exponent describes the critical properties along the entire phase boundary in Fig. 1(b), except the terminal points. For the anisotropic case $\left(p_{1} \neq p_{3}\right)$ we obtain (for the first time as far as we know) a different universality class, characterized by $\beta=0.5 \pm 0.02$.

In order to understand better the nature of the nonfrozen phase far from the deterministic corner $p_{1}$ 


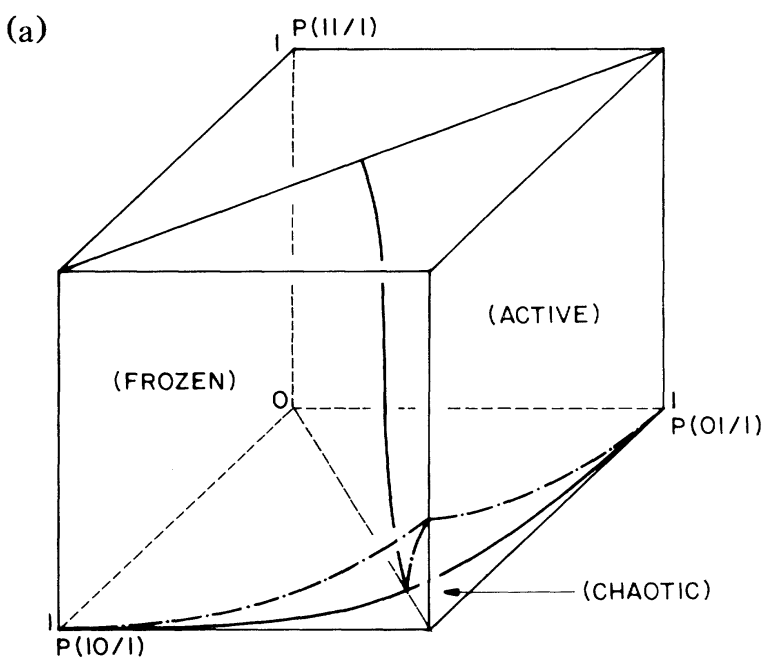

(b)

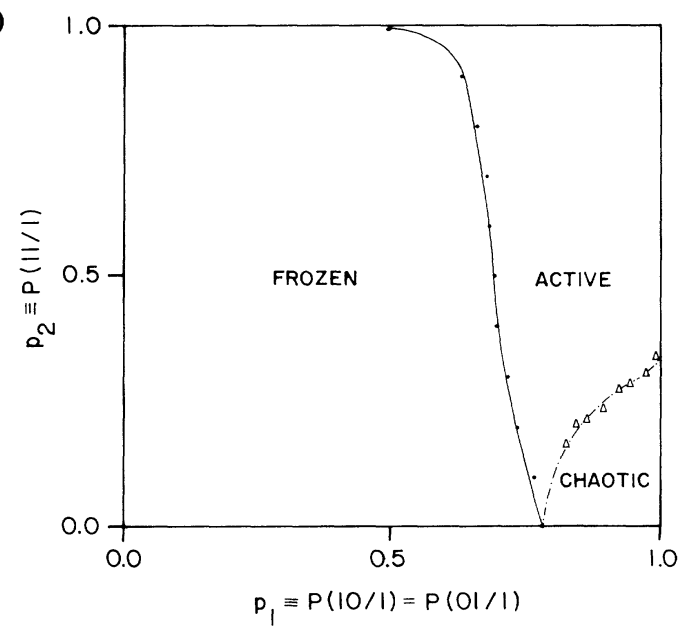

FIG. 1. (a) Phase diagram of the generalized DomanyKinzel PCA. The solid lines belong to the critical surface separating the frozen $(M=0$ and $\Psi=0)$ and active $(M \neq 0$ and $\Psi=0$ ) phases. The dashed lines belong to the boundary between the active and chaotic $(M \neq 0$ and $\Psi \neq 0)$ phases. (b) $P(10 / 1)=P(01 / 1)$ phase diagram. The data correspond to simulations with $N=3200$ sites; transients of 10000 (3000) time steps were used for the frozen-active (active-chaotic) phase transitions. The damage was averaged over another 3000 time steps.

$=p_{3}=1$ and $p_{2}=0$ (rule 90 in Wolfran's notation), we have studied how damage spreads throughout this PCA. To do this, we first simulate the automaton until it attains equilibrium. Then we make a replica of the system where we create an "initial damage" by flipping randomly a fraction $p$ of the sites. As $t$ evolves, the initial damage spreads through a damaged region where the sites in the two systems have different values. This damage is measured by a normalized Hamming distance $\Psi$ defined as the fraction of sites in the replica system that differ from their counterparts in the original system. In Fig. 2

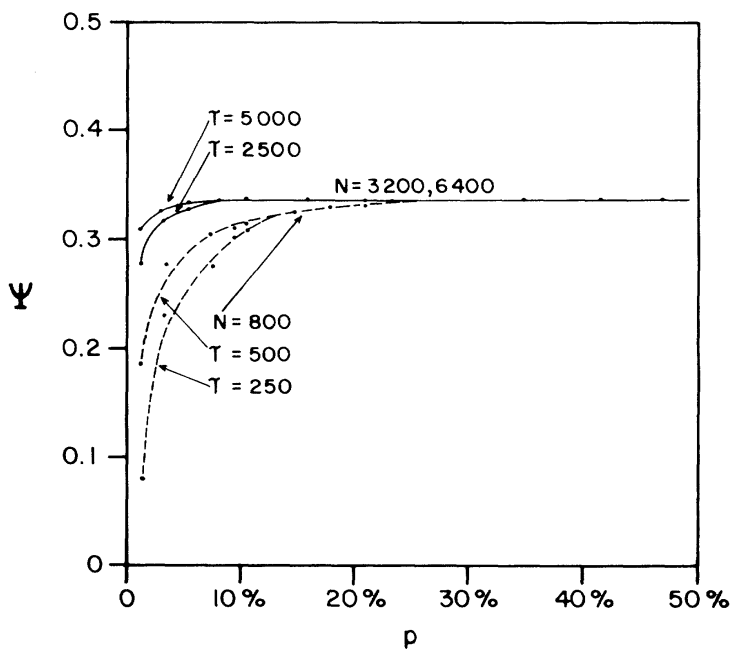

FIG. 2. The parameter $\Psi$ for different values of the initial damage probability $p$, for $P(11 / 1)=0.15$ and $P(10 / 1)$ $=P(01 / 1)=0.90$. The lines are guides to the eye. $\tau$ is the time (after arrival to equilibrium) over which $\Psi$ was averaged.

we show $\Psi$ as a function of the initial damage $p$ for different system sizes and evolution times. From these data we see that a fraction $p \simeq 50 \%$ is quite convenient for reliable simulations. If the damage spreads through the entire PCA the automaton is sensitive to the initial conditions (chaotic phase). Damage spreading is a powerful tool which has been used to study dynamical phase transitions in Ising and Q2R models, ${ }^{6}$ spin glasses, ${ }^{7}$ deterministic cellular automata, ${ }^{8}$ and other spin models such as the axial next-nearest-neighbor Ising and the $X Y$ models. ${ }^{9}$ Recently, Coniglio et al. ${ }^{10}$ showed an interesting exact relation between damage spreading and

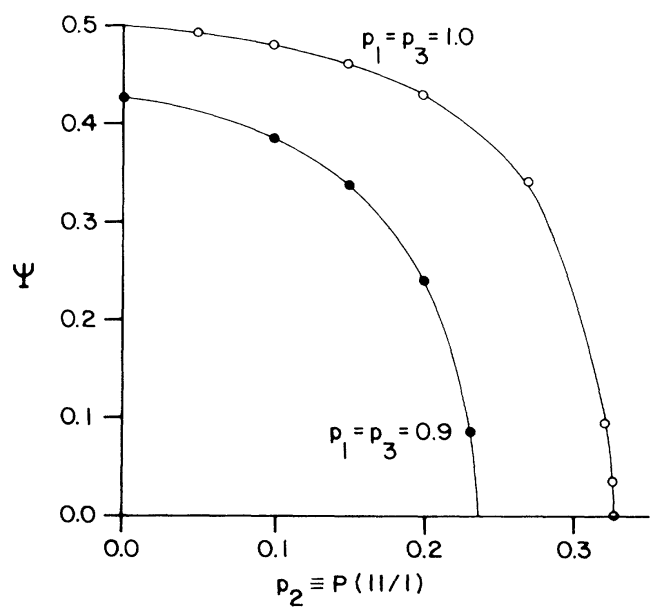

FIG. 3. The damage spread parameter $\Psi$ as a function of the probabilities. The data correspond to 3200 sites, transient until equilibrium of 2000 time steps, and damage evolution for another 3000 times steps. 
thermodynamical properties.

To calculate $\Psi$ we average over a period which is at least as long as the size of the chain. The parameter $\Psi$ vanishes continuously (see Fig. 3) on a critical surface (different from that associated with $M$ ) which also is indicated in Fig. 1 [this surface, which we denote by $\left(p_{1 s}, p_{2 s}, p_{3 s}\right)$, has been established by extrapolating the results corresponding to increasingly large CA sizes; in Fig. 2 we present typical values of $N$ and $p$ which guarantee that the $N \rightarrow \infty$ limit has practically been attained]. In the neighborhood of the deterministic point $\left(p_{1}, p_{2}, p_{3}\right)=(1,0,0)$ the damage does not die out, but it moves over the whole lattice. However, it remains small and vanishes in the thermodynamic limit $N \rightarrow \infty$. As a function of $\left(p_{1}, p_{2}, p_{3}\right) \Psi$ vanishes according to the power law $\Psi=\left(p_{2 c}-p_{2}\right)^{\mu}$, with $\mu=0.37 \pm 0.10$ for the isotropic case (excepting possibly for the terminal points); the error bar 0.10 has been adopted similarly to the error bar of $\beta$. We have not checked but it would not be surprising if $\mu$ were different for $p_{1} \neq p_{3}$.

In summary, we studied the phase diagram of the anisotropic Domany-Kinzel-like stochastic one-dimensional cellular automaton. In addition to the two known phases (frozen and active, i.e., an attractor which presents respectively a fixed-point structure and extremely long cycles) detected by studying the behavior of the order parameter $M$, we found a third phase (chaotic; $M \neq 0$ and $\Psi \neq 0$ ) related to the spread of damage (more precisely, presenting simultaneously extremely long cycles and sensitivity to the initial conditions). The critical surfaces where $M$ and $\Psi$ vanish are different except on the $p_{2}=0$ plane where they appear to share one and the same critical line.

${ }^{(a)}$ Permanent address: Departamento de Física, Universidade Federal de Viçosa, 36570 Viçosa, Minas Gerais, Brazil.

'Physica (Amsterdam) 10D (1984), Vol. 1.

${ }^{2}$ S. Wolfram, Theory and Applications of Cellular Automata (World Scientific, Singapore, 1986).

${ }^{3}$ P. Rujan, J. Stat. Phys. 49, 139 (1987); A. Georges and P. Le Doussal, J. Stat. Phys. 54, 1011 (1989).

${ }^{4}$ E. Domany and W. Kinzel, Phys. Rev. Lett. 53, 447 (1984); W. Kinzel, Z. Phys. B 58, 229 (1985).

${ }^{5}$ N. I. Jaeger, K. Möller, and P. J. Plath, J. Chem. Soc., Faraday Trans. 1, 82, 3315 (1986); P. J. Plath, Catal. Today 3, 475 (1988); P. J. Plath, K. Möller, and N. I. Jaeger, J. Chem. Soc., Faraday Trans. 1, 84, 1751 (1988).

${ }^{6}$ H. Eugene Stanley, Dietrich Stauffer, János Kertész, and H. J. Herrmann, Phys. Rev. Lett. 59, 2326 (1987); U. M. S. Costa, J. Phys. A 20, L583 (1987); B. Derrida and G. Weisbuch, Europhys. Lett. 4, 657 (1987); A. U. Newmann and B. Derrida, J. Phys. (Paris) 49, 1647 (1988).

${ }^{7}$ L. De Arcangelis, A. Coniglio, and H. J. Hermann, Europhys. Lett. 9, 749 (1989); L. De Arcangelis, H. J. Herrmann, and A. Coniglio, J. Phys. A 22, 4659 (1989).

${ }^{8}$ L. R. da Silva, A. Hansen, and S. Rony, Europhys Lett. 8, 47 (1989).

${ }^{9}$ Michael N. Barber and B. Derrida, J. Stat. Phys. 51, 877 (1988); D. Golinelli and B. Derrida, J. Phys. A 22, L939 (1989).

${ }^{10}$ A. Coniglio, L. De Arcangelis, H. J. Herrmann, and N. Jan, Europhys. Lett. 8, 315 (1989). 